\title{
RADIATION OF HEAT IN AN INSULATING VACUUM LAYER
}

\author{
MÁRIA MinÁrová
}

\begin{abstract}
The paper is motivated by the previous research concerning the heat transfer in a heat accumulation device. The device had been explored, built up and tested with the aim of utilization of cheap solar energy and its storage. In this heat storage system, a vacuum-like gap between two concentric containers acts as an insulating layer, radiation being the predominant heat transfer type in the gap. The better knowledge and understanding of the heat exchange by radiation, the more effectiveness of the insulation of the layer can be reached. Heat transfer by radiation is explored in the paper, mathematical model is set up, the algorithm of non-linear transient computation is introduced, and some illustrative results of this computation are performed.
\end{abstract}

\section{Introduction}

Nowadays we live in an age of saving energy effort, in the sustainable and renewable energy utilization necessity. The extensive insulation is realized, the solar, wind and geothermal energy is utilized instead of the fossil energy source. The timely question arises how to store the thermal energy acquired in the period of abundance with perspective of its exploitation in the date of its lack. Nevertheless, between the two season (summer and winter) several months pass and it is essential to ensure minimal heat loss at the lowest possible operating costs during this time. Therefore the accumulation tanks with the various insulating are used. An accumulation tank with double concentric containers with a vacuum-like layer between is discussed bellow. The inner, smaller container is placed inside the outer, bigger one being superimposed on four small wooden pedestals. Such a tank was built up, theoretically investigated and tested by a group of researchers from our university, see Figure 1 and [6].

(C) 2017 Mathematical Institute, Slovak Academy of Sciences. 2010 Mathematics Subject Classification: 35Q79, 65M60, $65 \mathrm{Z} 05$.

Keywords: insulation gap, transient heat equation, heat radiation, nonlinearity, heat flux, heat accumulation, view factor, temperature field.

This work was supported by grant VEGA 1/0456/17 and VEGA 1/0696/14. 
Herein, the thermal aspect is regarded. The high effectiveness of the vacuum or thin air is exploited. For the sake of the best utilization of the insulation capability of the vacuum (specially vacuum-like layer of certain geometry, with certain enclosing surface properties), knowing the functioning of the radiation as the heat transfer type is essential and to learn how it works within the configuration of the accumulation device. And this is the motivation for the investigation described in the paper.

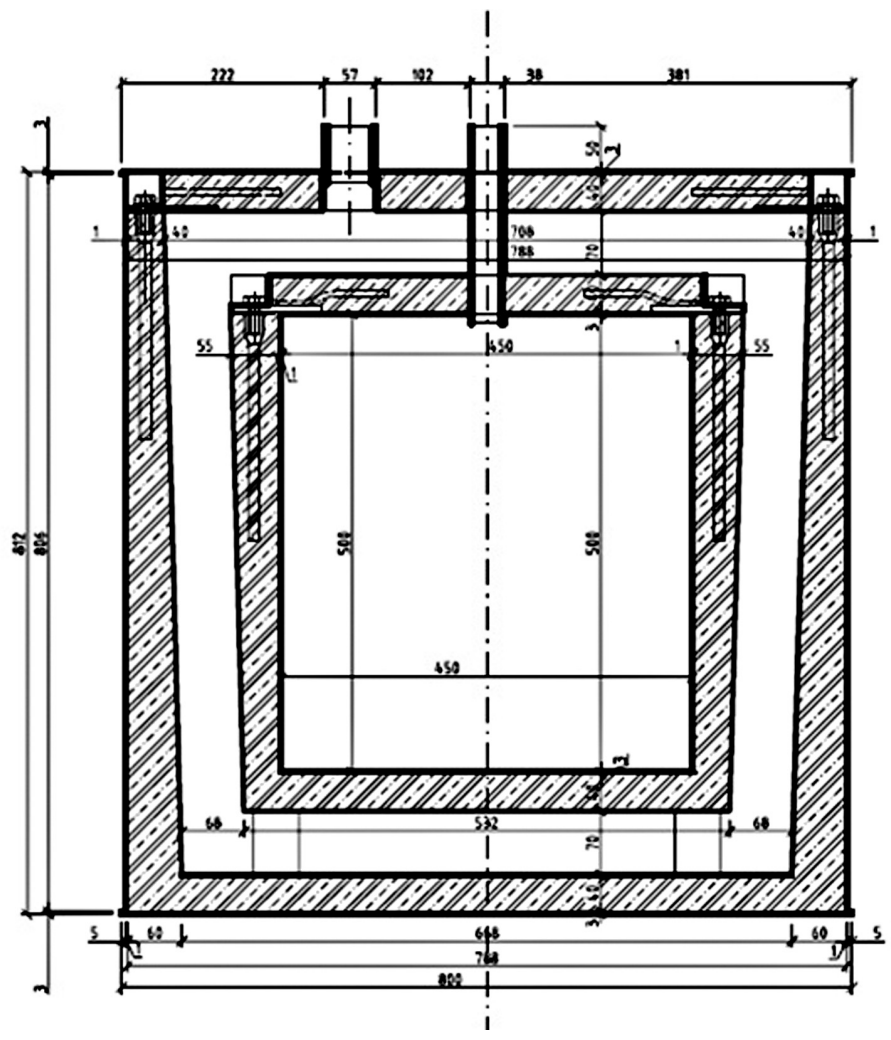

Figure 1. Axial section of the heat accumulation tank 6].

The paper deals with the heat radiation and its various aspects. Here, the view factor is worth emphasizing. As it strongly influences the magnitude of the transferred heat, its correct stipulation is essential. The heat transfer is treated by using the finite element method that operates on the domain divided into finite elements. The computation of view factor itself is performed after the meshing of the domain is accomplished and prior to the transient thermal analysis execution. Each facet exposed to the radiation (enfolding the enclosure where the radiation takes place) is involved in the view factor calculation. 
Since the view factor depends on the configuration of the surfaces, each pair of facets (one releasing - one receiving heat) is treated individually, just the projections of the radiation rays to outer normals of facets are taken into account. Finally, all data are collected in the view factor matrix ready for further thermal analysis. And this is the crucial point of the computation, the main contribution to the investigation concerning heat exchange throughout the accumulation device and the surroundings, the main point of the paper. The special section is devoted to the view factor calculation.

The material of the two containers is concrete. Hence, there were some initial technical problems that had to be overcome even before the realization, e.g., stiffness enforcement was inevitable as the high pressure difference on the both sides of each containers is expected, unwanted water penetration through concrete due to the pressure difference should be prevented, airtightness of the gap between containers has to be ensured, see [1], [3]. As it can be seen in Figure 1, on the upper part of the container there is a pipe leading to the very inner space of the container, the pipe that the hot water is pumped through and the other one where the sucking vacuum device is attached. After the hot water is poured into the tank and the air is sucked out from the inter-space, both pipes are removed and the holes clogged up. This initial stage is the starting point of the theoretical investigation.

\section{Radiation and its involvement in transient heat transfer}

\subsection{Heat transfer governing equation, initial-boundary value problem}

The domain of investigation is depicted in Figure 2. The initial thermal states are following: We suppose the initial temperature of $90^{\circ} \mathrm{C}$ in the heat accumulation medium (water) and in the inner container, and $12{ }^{\circ} \mathrm{C}$ elsewhere, including the surroundings where the accumulation tank is placed. This is the instant the cooling starts, the instant we begin to compute/observe the temperature field evolution in the entire accumulation device within next thirty days. As we expect that the evolving of the temperature field will proceed gradually and calmly, we can neglect the heat convection due to movement of the water. Hence, the heat both in the liquid and in the solid parts of the device flows in the opposite direction to the thermal gradient, governed by the transient heat equation

$$
c(X, t) \rho(X, t) \frac{\partial T(X, t)}{\partial t}=\nabla(\lambda(X, t) \nabla T(X, t)),
$$

where $T(X, t)\left[{ }^{\circ} \mathrm{C}\right]$ is temperature at spatial point $X$ in time $t, c(X, t)[\mathrm{J} /(\mathrm{kgK})]$ specific heat, $\rho(X, t)\left[\mathrm{kg} / \mathrm{m}^{3}\right]$ density of material, $\lambda(X, t)[W /(m K)]$ heat conduction coefficient. Due to rotational symmetry after the sucking pump and the inlet 


\section{MÁRIA MINÁROVÁ}

tube removal - the two short pipes upon the device in Figure 1 and clogging the remaining holes, we can treat the entire heat transfer as a $2 \mathrm{D}$ axisymmetric taking whichever half axial section as the representative 2D domain, see Figure 2.
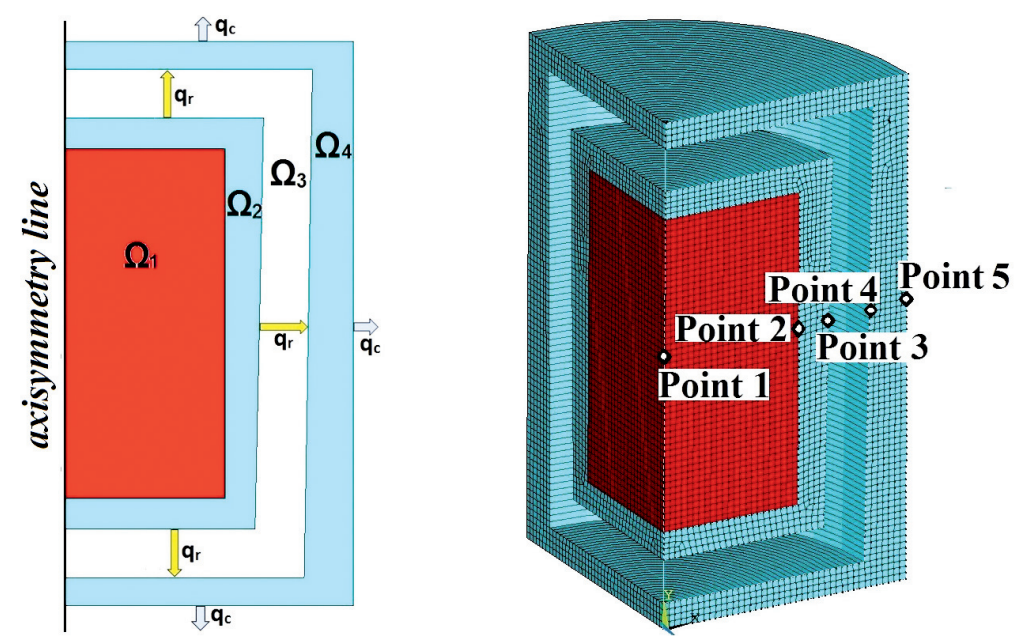

Figure 2. The domain of investigation. Left: characteristic 2D axial halfsection of axisymmetric body, radiation and convection fluxes are highlighted. Right: five points marked, where the temperature is traced.

The heat transfer is driven by (10) in $\Omega_{1}, \Omega_{2}$ and $\Omega_{4}$, the radiation takes place in $\Omega_{3}$ reading the Stefan-Boltzmann law

$$
q_{r}=\sigma \varepsilon F\left(T_{1}^{4}(X, t)-T_{2}^{4}(X, t)\right)
$$

with $q_{r}\left[W / m^{2}\right]$ being radiative heat flux per unit area, $\sigma\left[W /\left(m^{2} K^{4}\right)\right]$ Boltzmann constant of value $5.670410^{-8}, \varepsilon[-]$ emissivity of material, $T_{1}(X, t), T_{2}(X, t)[K]$ both - hot and cold surface temperatures (variable in time), $F[-]$ view factor. View factor is very important parameter in the radiation process. Its magnitude affects the radiation proceeding significantly. The influence of geometry and stipulation of the view factor are described below in the subsection View factor (form factor) specification.

The conductive contribution, though very small of the thin air in the gap, i.e., in subdomain $\Omega_{3}$, see Figure 2 Left, is inclusive. On the outer boundary of the domain, the convection heat exchange between the wall and air proceeds

$$
q_{c}=h_{c}\left(T(X, t)-T_{b u l k}\right)
$$

with $q_{c}\left[W /\left(m^{2}\right)\right]$ being convective heat flux per unit area, $h\left[W /\left(m^{2} K\right)\right]$ film coefficient on outer wall of the tank, $T_{b u l k}[K]$ bulk temperature of ambient, where the tank is kept. 
Finally, the IBVP on the entire domain $\Omega$ is accomplished by imposing the initial temperature distribution $90^{\circ} \mathrm{C}$ in $\Omega_{1} \cup \Omega_{2}$ and $12^{\circ} \mathrm{C}$ in $\Omega_{3} \cup \Omega_{4}$ (stepped function on $\Omega$ )

$$
\begin{aligned}
& T(X, 0)=90^{\circ} \mathrm{C} \quad \text { in } \Omega_{1} \cup \Omega_{2}, \\
& T(X, 0)=12{ }^{\circ} \mathrm{C} \quad \text { in } \Omega_{3} \cup \Omega_{4} .
\end{aligned}
$$

\subsection{Radiation between surfaces}

Heat radiation is the specific kind of heat transfer via electromagnetic waves, the wavelength of which varies within the range $0.75-400 \mu m$. The waves propagate in a space at the speed of light without having need of a bearing substance for the propagation. Its magnitude is determined by the thermal stage of the participating bodies (temperature, radiosity, emissivity, reflexivity), by the geometry configuration (shapes, angles, segmentivity) and the quality of the surfaces involved in radiation (smoothness, silkiness, color). The radiative energy $Q_{0}$ striking a surface of a body ("grey body") either goes through the body $Q_{T}$, either is reflected by the surface $Q_{R}$ or absorbed by the body $Q_{A}$; $Q_{0}=Q_{T}+Q_{R}+Q_{A}$, see Figure 3. Dividing this equation by $Q_{0}$ we obtain the splitting ratio equation for the radiation

$$
1=T_{r}+R_{r}+A_{r}
$$

where the numbers $T_{r}, R_{r}, A_{r} \in[0,1]$ are the coefficients of transmittance, reflexivity and absorptivity, respectively [4. At the specific temperature, the perfect black-body (ideal one) has the maximal possible radiative ability, $A_{r}=1$ having zero reflexivity and zero transmissivity at the same time. If $R_{r}=1$, it is a perfect mirror or prefect white body, $T_{r}=1$ - diathermic body. The most of materials, also all that involved in our model, are opaque so the transmittance can be neglected, hence we use (5) in the form

$$
1=R_{r}+A_{r} .
$$

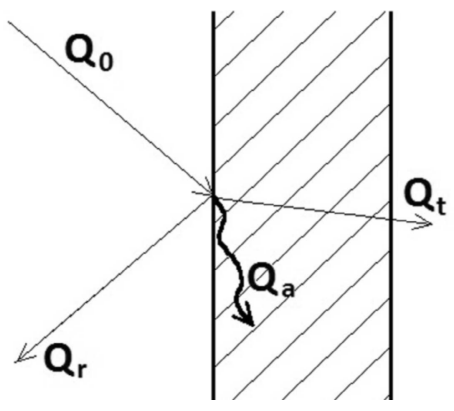

FiguRE 3. Radiation $Q_{0}$ decomposition to reflected $Q_{R}$, absorbed $Q_{A}$ and transposed $Q_{T}$. 


\section{MÁRIA MINÁROVÁ}

The radiation in subdomain $\Omega_{3}$ operating on its enclosing surfaces is stated as the boundary condition (2) on that surfaces, see Figure 2, Left.

In the following we will suppose the unique wavelength of the thermal radiation and the temperature independent physical parameters of matters involving in the process of heat transfer. Moreover, the materials are considered as homogeneous and isotropic, so all thermal properties are constant. The Stefan-Boltzmann law (2) is the governing equation of the radiation.

\subsection{Flow balance on the surfaces enclosing $\Omega_{3}$}

First let us observe the heat exchange on the outer boundary of inner, hot container. The heat exchange between insulating gap and inner concrete container and realized on its surface is represented by the flow balance between the conductive and radiative fluxes

$$
q_{v}=q_{r}
$$

This means that the conductive heat flux $q_{v}$ outgoing from the hot container through its surface is equal to the radiative flux $q_{r}$ transporting the heat energy farther outwards from the surface in the direction of outer normal. Taking (1) that governs the heat flow in the homogeneous concrete container into the consideration, the first time step temperature change on the surface can be approximated by the equation

$$
\frac{{ }^{1} T_{2}-{ }^{0} T_{2}}{{ }^{1} \Delta t}=\frac{\lambda}{c \rho} \nabla^{2} T,
$$

where ${ }^{0} T_{2},{ }^{1} T_{2}$ are the surface temperatures at the beginning and at the end of the time step, respectively, ${ }^{1} \Delta t$ is the initial time step length. Further, (8) can be rearranged by using the Fourier law of heat conduction $q_{v}=-\lambda \nabla T$,

$$
{ }^{1} T_{2}={ }^{0} T_{2}-\left({ }^{1} \Delta t\right) \frac{1}{c \rho} \nabla\left({ }^{0} q_{v}\right),
$$

where the initial heat flux ${ }^{0} q_{v}$ is the conductive heat flux outgoing from the concrete inner container to the gap. Let us recall that homogeneity and isotropy of material yield the constant $c, \rho, \lambda$ herein. In (9) the conductive heat flux is unknown, but due to flow balance (7) it can be replaced by ${ }^{0} q_{r}$ which is known and can be incorporated into (9) in the form of (2)

$$
{ }^{1} T_{2}={ }^{0} T_{2}+\left({ }^{1} \Delta t\right) \frac{1}{c \rho} F \varepsilon \sigma\left({ }^{0} T_{2}^{4}-{ }^{0} T_{1}^{4}\right) .
$$

Let us summarize the thermal process on the warmer container outer surface releasing the radiation with ${ }^{0} T_{2}$ is the initial temperature and ${ }^{1} T_{2}$ computed value)

$$
{ }^{0} T_{2} \rightarrow{ }^{0} q_{r} \rightarrow{ }^{0} q_{v} \rightarrow{ }^{1} T_{2}
$$




\section{RADIATION OF HEAT IN AN INSULATING VACUUM LAYER}

Accordingly, the similar process runs on the inner surface of outer container (cold one) of the initial temperature ${ }^{0} T_{1},{ }^{1} T_{1}$ being the requested value, the resulting temperature of the surface at the end of the time step

$$
{ }^{0} T_{1} \rightarrow-{ }^{0} q_{r} \rightarrow{ }^{0} q_{v} \rightarrow{ }^{1} T_{1} .
$$

Here the sign minus in front of the flux means the heat propagates from the insulating gap to the concrete outer container. At the beginning of the cooling process, as the difference ${ }^{0} T_{2}^{4}-{ }^{0} T_{1}^{4}$ is too high, the radiative flux is changing rapidly in time. So, to avoid the heavy errors, we have to proceed in time just with small steps when tracing the thermal process. Gradually, as the radiative flow decreases, the time steps can be appropriately enlarged. The length of the time increment is set within the iterative process. A starting value is assessed first (set by user or the default value taken). Then the flux and resulting temperatures are computed. Later on, the actual time interval is shorten twice, the new computation for the two subintervals is executed and the comparison of the both solution is done. If the sufficient accordance is reached, the starting time step is taken and the next time step is realized. This process of finding the length of the first (each further) time step can be written in the form of pseudo-code:

1. [initial temperatures of both cold/hot surfaces] ${ }^{0} \mathbf{T}_{\mathbf{c}},{ }^{\mathbf{0}} \mathbf{T}_{\mathbf{h}}$,

2. [radiative flux computed by using (2) $]^{\mathbf{0}} \mathbf{q}$,

3. [starting time step value - either default or set by user] ${ }_{1}^{1} \boldsymbol{\Delta} \mathbf{t}$,

4. [temperatures on both - cold and hot surfaces at the endpoint of time step from (10)] ${ }_{1}^{1} \mathbf{T}_{\mathbf{c}},{ }_{1}^{1} \mathbf{T}_{\mathbf{h}}$,

5. [interpolated values] ${ }_{\frac{1}{2}}^{1} \tilde{\mathbf{T}}_{\mathbf{c}}, \frac{1}{2} \tilde{\mathbf{T}}_{\mathbf{h}}, \frac{1}{2} \mathbf{\mathbf { q }}$,

6. [time step length shortened] ${ }_{\frac{1}{2}}^{1} \Delta \mathbf{t}$,

7. [computed values at half time step] ${ }_{\frac{1}{2}}^{1} \mathbf{T}_{\mathbf{c}},_{\frac{1}{2}}^{1} \mathbf{T}_{\mathbf{h}}, \frac{1}{2} \mathbf{q}$,

8. [testing criterion of finishing]

$$
\left|\frac{1}{\frac{1}{2}} \mathbf{T}_{\mathbf{c}}-\frac{1}{2} \tilde{\mathbf{T}}_{\mathbf{c}}\right|<\mathbf{c}_{\mathbf{t s t}} \text { and } \quad\left|\frac{1}{\frac{1}{2}} T_{h}-{ }_{\frac{1}{2}}^{1} \tilde{T}_{h}\right|<h_{t s t} .
$$

Remark. Useful information about pseudo-codes can be found, e.g., in [2].

The length of the time increment length with lapsing iterations as appropriate in computation within described mathematical model, is depicted in Figure 4.

\subsection{View factor (form factor) specification}

Generally speaking, the form factor quantifies the ratio of the amount of radiation striking the receiving surface and amount of radiation released from the emitting surface. The angles between the ray and the normals to both surfaces 


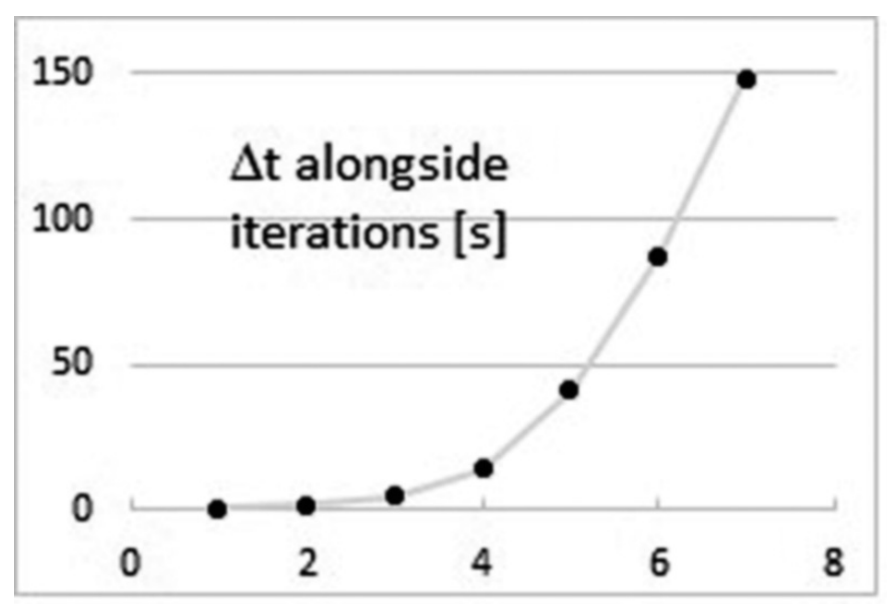

Figure 4. Time increment length in the first iterations.

play the significant role here, see Figure 6. Finally, the entire form factor of radiation is the sum of the view factors of particular pairs of surface segments

$$
F=\sum_{k=1}^{n} \sum_{l=1}^{m} F_{k l}
$$

with

$$
F_{k l}=\frac{1}{A_{k}} \int_{A_{k}} \int_{A_{l}} \frac{\cos \varphi_{k} \cos \varphi_{l}}{\pi r^{2}} d A_{l} d A_{k},
$$

where $F_{k l}$ is the form factor influencing the radiation which propagates from the $k$ th surface segment towards the $l$ th one, $\varphi_{k}$ is the angle between the radiation ray and the outer normal of the $k$ th segment, (likewise $\varphi_{l}$ ), $r$ is the length of the ray between both surface facets.

In case of segmented surfaces, each pair of surface segments (one from receiving, one from releasing surface), see Figures 5 and 6 , has to be treated separately. Physically spoken, only the perpendicular projection to the normal vector of the radiation is taken into account, see Figure 6.

\subsection{Flow balance on the surfaces enfolding the vacuum - like layer, absorptivity and reflexivity operating}

As mentioned above, on the interface between the inner container and insulating gap the outcoming heat conducted from the inside of the concrete material is equal to the amount of the heat transferred into and through vacuum-like layer. The difference between the temperatures fourth powers on both surfaces is the driving force of the radiation. Herein, the ratio between reflexivity and absorptivity is essential, see (6). By optimal finishing of surfaces enclosing the 


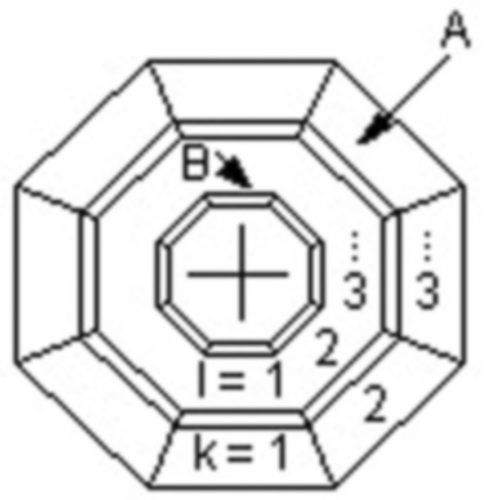

Figure 5. Two segmented surfaces A and B radiating one to another [5].

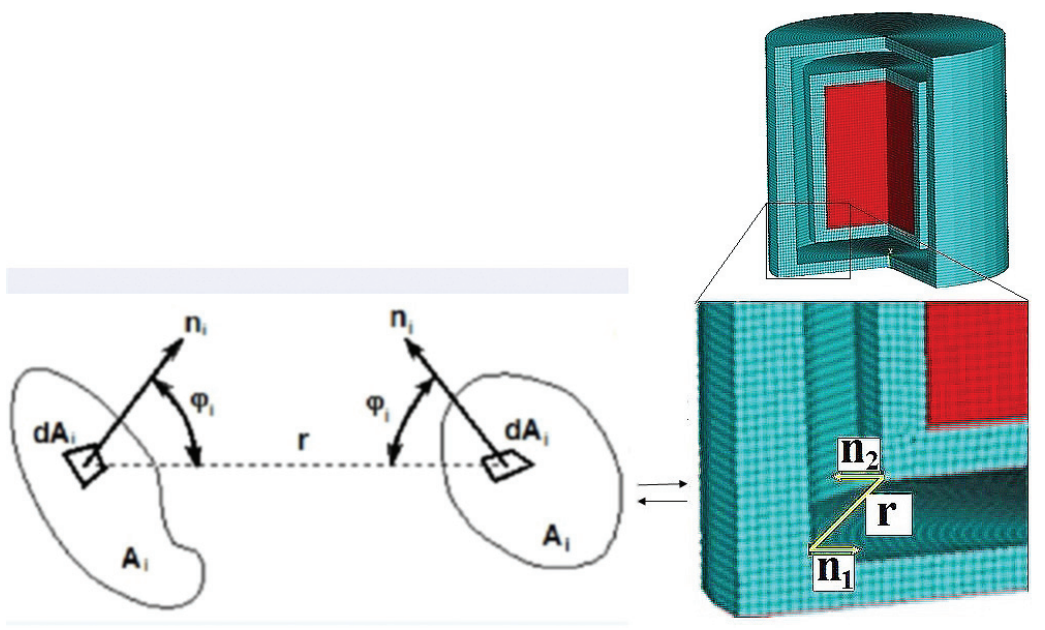

FiguRE 6. Radiation ray $r$ between two small areas $d A_{i}$ and $d A_{j}$; their normals' $\mathbf{n}_{1}$ and $\mathbf{n}_{2}$ directions, angles between normals and $r$; Left: generally, Right: on investigated tanker.

radiation domain (smoothness, brightness, glazing) the reflexivity of that surfaces grows while the absorptivity goes down. So the heat transfer through the gap is even more hindered. Let us trace the itineracy of the certain amount of the radiative energy $E_{1}$ emitted from a flat surface $S_{1}$ with absorptivity $A_{1}$ and reflexivity $R_{1}$ towards a parallel surface $S_{2}$ with absorptivity $A_{2}$ and reflexivity $R_{2}$ of the same area, supposing the energy being either absorbed or reflected 


\section{MÁRIA MINÁROVÁ}

$\left(S_{1}: 1=A_{1}+R_{1}\right.$ and $\left.S_{2}: 1=A_{2}+R_{2}\right)$ :

$S_{1}$ emits $E_{1}$,

$S_{2}$ absorbs $A_{2} E_{1}$

$S_{2}$ reflects $\left(1-A_{2}\right) E_{1}$,

$S_{1}$ absorbs $A_{1}\left(1-A_{2}\right) E_{1}$,

$S_{1}$ reflects $\left(1-A_{1}\right)\left(1-A_{2}\right) E_{1}=B E_{1}$,

* denoting $B=\left(1-A_{1}\right)\left(1-A_{2}\right)$,

$S_{2}$ absorbs $A_{2}\left(1-A_{1}\right)\left(1-A_{2}\right) E_{1}=A_{2} B E_{1}$,

$S_{2}$ reflects $\left(1-A_{2}\right)\left(1-A_{1}\right)\left(1-A_{2}\right) E_{1}=\left(1-A_{2}\right) B E_{1}$,

$S_{1}$ absorbs $A_{1}\left(1-A_{2}\right)\left(1-A_{1}\right)\left(1-A_{2}\right) E_{1}=A_{1}\left(1-A_{2}\right) B E_{1}$,

$S_{1}$ reflects $\left(1-A_{1}\right)\left(1-A_{2}\right) B E_{1}=B^{2} E_{1}$, etc.

Overall, $S_{1}$ releases $\left(1+B+B^{2}+\cdots+B^{n}+\cdots\right) E_{1}$ and receives back $\left(1-A_{2}\right)\left(1+B+B^{2}+\cdots+B^{n}+\cdots\right) E_{1}$ when the view factor $=1$ is supposed. Otherwise, each item should be multiplied by the corresponding view factor.

Remark. The particular view factors within the computation are collected in so-called view factor matrix. Its fragment can be seen in Figure 7.

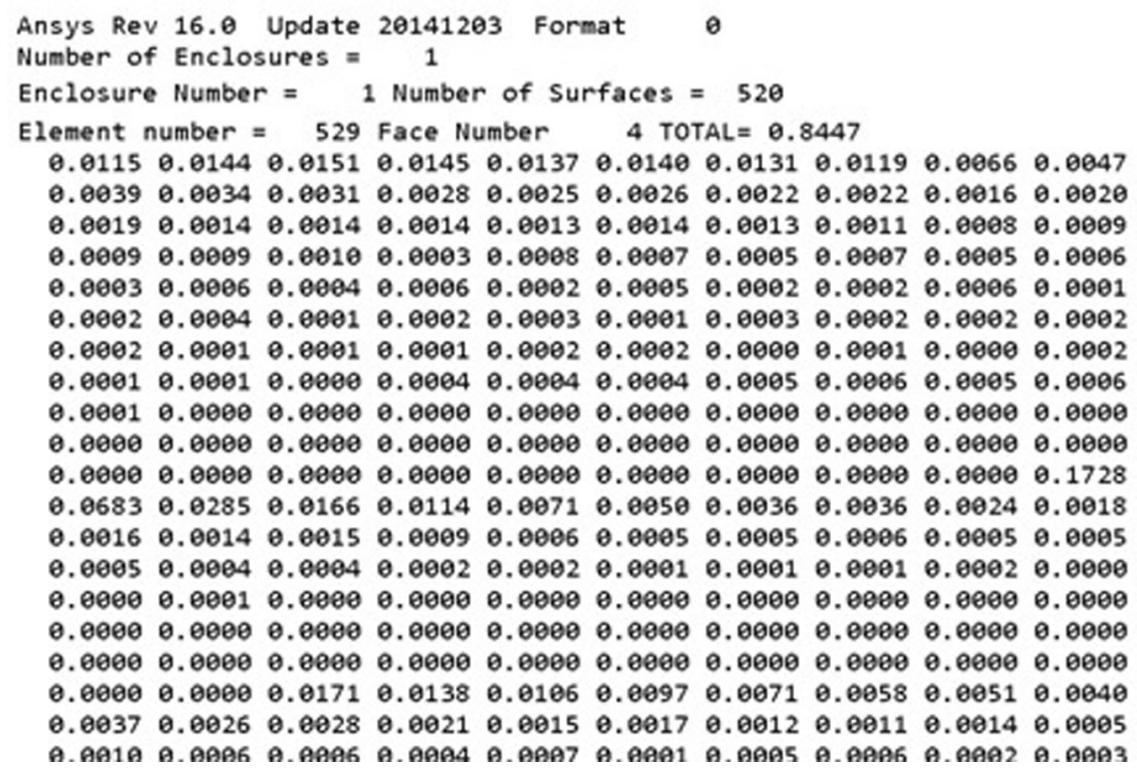

FIGURE 7. Fragment of view factor matrix as completed within the program.

It is important to emphasize the reflexivity power as the essential hindering mechanism of the heat transfer process. As mentioned before, reflexivity can be increased by suitable finishing (smoothing, polishing, glazing, painting) of the surfaces enclosing vacuum gap. 


\section{Illustrative results of the finite element computation}

The computation of the explored algorithm based on the gathered knowledge as described above, was realized within the finite element software. A built-in method for the view factor matrix was used, taking the facets of particular finite elements as the surface segments. The axisymmetric 2D model was used, nonlinear transient analysis was carried out. In Figure 8 and Figure 9, the thermal fluxes and thermal gradients are depicted. Both figures the strong insulating

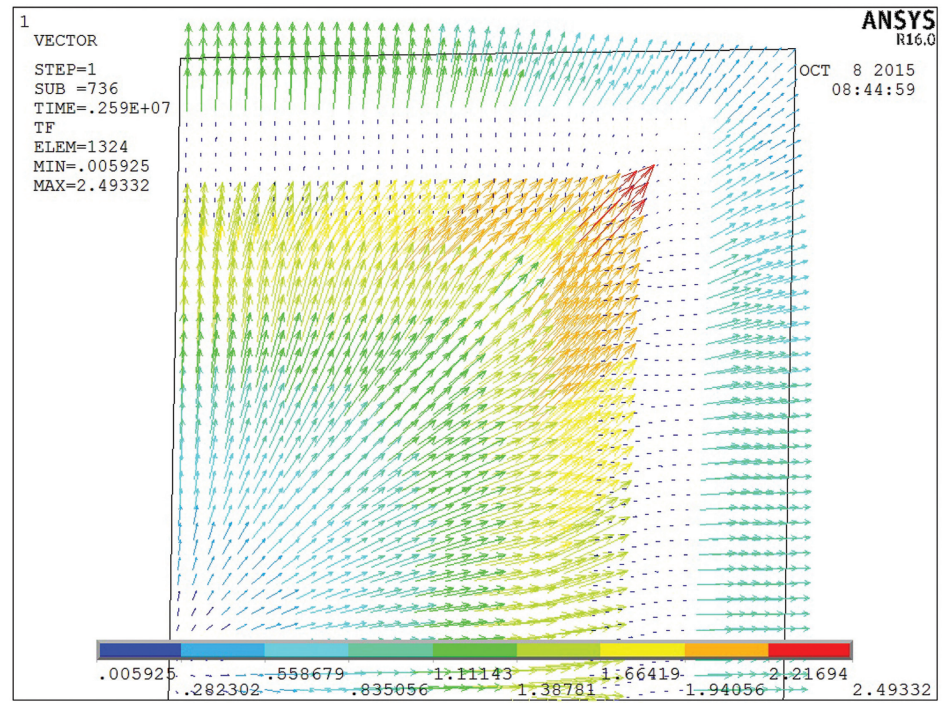

FiguRE 8. Vector field of thermal fluxes in 2D section.

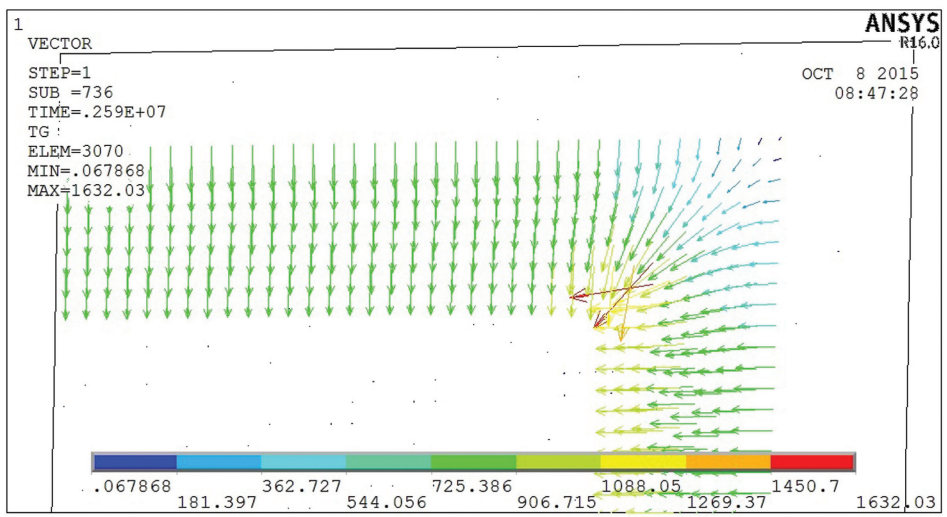

FiguRE 9. Vector field of temperature gradients in $2 \mathrm{D}$ section. 


\section{MÁRIA MINÁROVÁ}

power of the vacuum-like gap. While the temperature gradients are high in the gap, the thermal fluxes are very small there. That is why the isotherms are dense therein, see Figure $10 \mathrm{~b}$ ). For the sake of radiation proceed observing, the five points are imposed lying on the horizontal line leading through the very middle of the device where the maximal values are expected. In these points the temperature course is traced during the time interval. The first point of the set is situated in the very middle, the others on all interfaces along the horizontal outward direction, see Figure 2, Right.
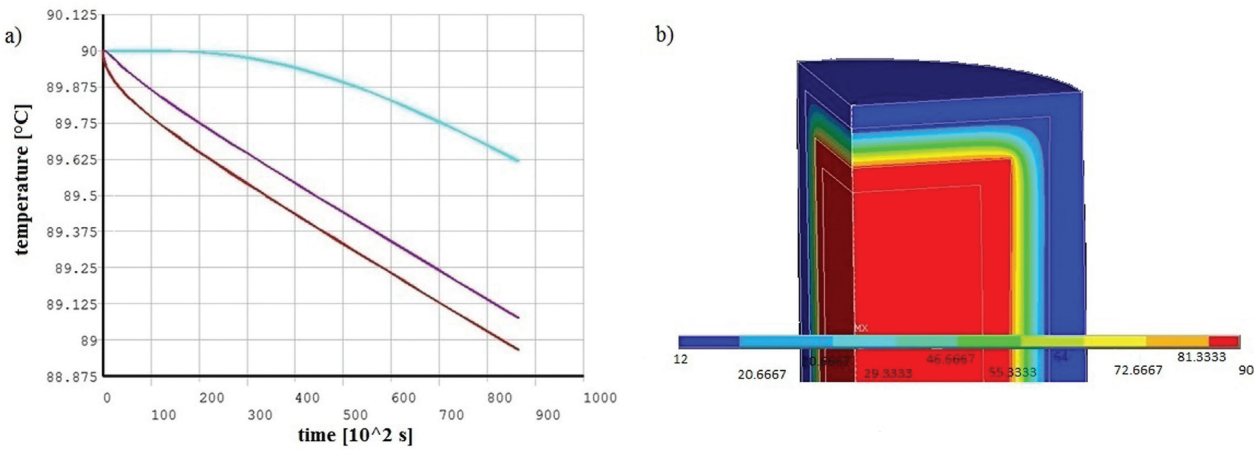

Figure 10. a) Temperature course in Point 1 - upper curve, Point 2 -middle curve, Point 3 - lower curve, b) temperature field in the entire domain after 24 hour of cooling.
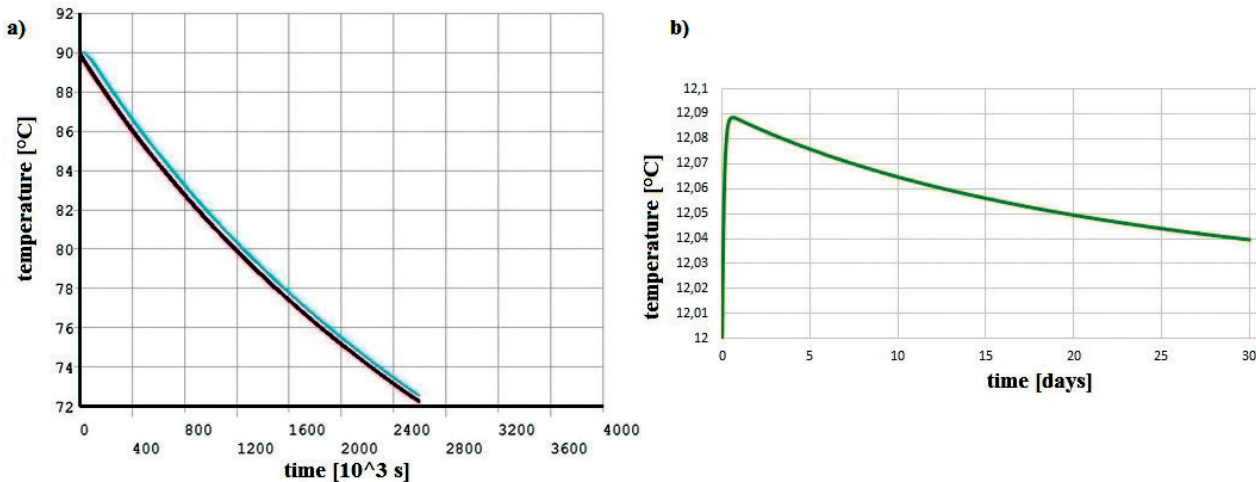

FiguRE 11. a) Temperature course in the water and on the inner container surfaces during 30 days of cooling, b) temperature of the very outer surface within the same period. 


\section{RADIATION OF HEAT IN AN INSULATING VACUUM LAYER}

The temperature course in the Points $1-3$, see Figure $10 \mathrm{a}$ ), illustrates the cooling process in the first 24 hours in these places. The influence of the initial conditions is evident here. The delay of the temperature decrease in the midpoint of the water, Point 1, documents this fact best. As expected, after this first phase of temperature field evolution, the temperatures of any place situated within the vacuum insulation descend alike, see also Figure 11 a), the cooling process during one month. Due to high heat conduction ability of concrete Point 2 and Point 3 have almost the same temperature course.

The illustration of the very good heat accumulation ability of the accumulation device is apparent from the temperature course on the outer boundary of the tank, Figure $11 \mathrm{a}$ ). Even though the temperature difference between the water and the surrounding of the accumulation tank persists to be high within the long period, the outer boundary of the tank is heated up just slightly, see Figure $11 \mathrm{~b}$ ).

\subsection{Result data interpretation, accumulated heat potential}

The data obtained in the finite elements analysis should be interpreted properly. As mentioned above, the set of points are of the maximal temperature within the particular verticals. Such a data are useful for the comparison with the experiments, as the temperature detecting device is obviously fastened on a surface. But the overall amount of the accumulated (and not lost so far) heat should be done by a kind of summation or integration. Remembering the fact that a 2D axisymmetric representation is taken into account, in the case of analytical expression of the temperature field just the volume integral of temperature function (a number) could represent the quantification of the overall accumulated heat potential. But in case of grid or element solution, the weighted sum should be realized instead (the weight $=$ the volume of the particular ring over the entire volume).

\section{Conclusion}

The paper is devoted to the radiation heat transfer. It deals with a theoretical study of physical background, it describes the building up of the physical and mathematical model of the real thermal device where the radiation plays the decisive role; the solving algorithm provided. The investigation arose from the practice and both theoretical exploration and numerical results are aimed to the practice. Various mathematical tools for the very practical purpose within the interdisciplinary investigation went through the entire process of investigation. The nonlinear transient thermal model is fully parametrized and can be used widely on accumulation tanks of the various geometry with various physical properties, including the finishing of the surfaces enclosing the insulating vacuum-like gap. 


\section{MÁRIA MINÁROVÁ}

\section{REFERENCES}

[1] BILČÍK, J.-SONNENSCHEIN, R.: Analysis of concrete water permeability, Projekt -Stavba, SKSI 1 (2015), 10-12.

[2] CHEnEY, W.-KINCAID, D.: Numerical Mathematics and Computing (5th ed.). Thomsom Learning, Austin, Texas, 2004.

[3] FILLO, L.: Development of methods for construction of thermal vacuum insulated containers, Projekt-Stavba, SKSI 1 (2015), 6-8.

[4] HALAhYJA, M.-FEHÉR, J.-HYKŠ, P.: Building thermal technology, lighting and acoustics (3rd ed.). Alfa, Bratislava, 1970.

[5] ANSYS, Theory Reference, Ansys documentation service, Vol. 5.6, 2015. http://research.me.udel.edu/ lwang/teaching/MEx81/ansys56manual.pdf

[6] JURIGOVÁ, M.-MINÁROVÁ, M.-CHMÚRNY, I.: Analysis of performance of thermal energy storage system, Appl. Mech. Materials 824, Scientific. Net (2016), 371-378.

Received April 13, 2017

Slovak University of Technology

Faculty of Civil Engineering

Radlinského 11

SK-810-05 Bratislava

SLOVAKIA

E-mail: maria.minarova@stuba.sk 